\title{
Improving the reliability of female fertility breeding values using type and milk yield traits that predict energy status in Australian Holstein cattle
}

\author{
O. González-Recio, ${ }^{*} \dagger \ddagger$ M. Haile-Mariam, ${ }^{*}{ }^{1}$ and J. E. Pryce ${ }^{*} \dagger \S$ \\ *Biosciences Research Division, Department of Economic Development, Jobs, Transport and Resources, Agribio, 5 Ring Road, Bundoora, \\ VIC 3083, Australia \\ †Dairy Futures Cooperative Research Centre, Bundoora, VIC 3083, Australia \\ ‡Instituto Nacional de Investigación y Tecnología Agraria y Alimentaria, Ctra. La Coruña km 7.5, 28040 Madrid, Spain \\ $\S$ La Trobe University, Bundoora, VIC 3083, Australia
}

\section{ABSTRACT}

The objectives of this study were (1) to propose changing the selection criteria trait for evaluating fertility in Australia from calving interval to conception rate at d 42 after the beginning of the mating season and (2) to use type traits as early fertility predictors, to increase the reliability of estimated breeding values for fertility. The breeding goal in Australia is conception within 6 wk of the start of the mating season. Currently, the Australian model to predict fertility breeding values (expressed as a linear transformation of calving interval) is a multitrait model that includes calving interval (CVI), lactation length (LL), calving to first service (CFS), first nonreturn rate (FNRR), and conception rate. However, CVI has a lower genetic correlation with the breeding goal (conception within $6 \mathrm{wk}$ of the start of the mating season) than conception rate. Milk yield, type, and fertility data from 164,318 cow sired by 4,766 bulls were used. Principal component analysis and genetic correlation estimates between type and fertility traits were used to select type traits that could subsequently be used in a multitrait analysis. Angularity, foot angle, and pin set were chosen as type traits to include in an index with the traits that are included in the multitrait fertility model: CVI, LL, CFS, FNRR, and conception rate at d 42 (CR42). An index with these 8 traits is expected to achieve an average bull first proof reliability of 0.60 on the breeding objective (conception within $6 \mathrm{wk}$ of the start of the mating season) compared with reliabilities of 0.39 and 0.45 for CR42 only or the current 5-trait Australian model. Subsequently, we used the first eigenvector of a principal component analysis with udder texture, bone quality, angularity, and body condition score to calculate

Received June 23, 2015.

Accepted September 16, 2015.

${ }^{1}$ Corresponding author: mekonnen.hailemariam@ecodev.vic.gov.au an energy status indicator trait. The inclusion of the energy status indicator trait composite in a multitrait index with CVI, LL, CFS, FNRR, and CR42 achieved a 12-point increase in fertility breeding value reliability (i.e., increased by $30 \%$; up to 0.72 points of reliability), whereas a lower increase in reliability ( 4 points, i.e., increased by 10\%) was obtained by including angularity, foot angle, and pin set in the index. In situations when a limited number of daughters have been phenotyped for CR42, including type data for sires increased reliabilities compared with when type data were omitted. However, sires with more than 80 daughters with CR42 records achieved reliability estimates close to $80 \%$ on average, and there did not appear to be a benefit from having daughters with type records. The cost of phenotyping to obtain such reliabilities (assuming a cost of $\mathrm{AU} \$ 14$ per cow with type data and $\mathrm{AU} \$ 5$ per cow with pregnancy diagnosed) is lower if more pregnancy data are collected in preference to type data. That is, efforts to increase the reliability of fertility EBV are most cost effective when directed at obtaining a larger number of pregnancy tests.

Key words: fertility, energy balance, type trait, selection index

\section{INTRODUCTION}

In recent decades, dairy cow fertility has been considered to be among the most important traits other than milk production (Bascom and Young, 1998; Groen et al., 1998). Female fertility is particularly important in countries that strive to match pasture growth to milk production. The extension of lactation by delaying pregnancy is not desirable in seasonal systems and entails a substantial economic loss (Bell et al., 2013). Nonetheless, no clear consensus exists as to which fertility traits must be included in selection indexes. Fertility is a complex trait with no single trait adequately describing it. Consequently, several measures of fertility are used to account for different aspects of the female 
reproductive cycle, such as showing estrus early in lactation, becoming pregnant in a timely way, and the ability to carry a pregnancy successfully to full term (Groen et al., 1998). Furthermore, every trait poses pros and cons in their use for genetic evaluations, such as censoring and skewed or discrete distributions, and are mostly management dependent (Oseni et al., 2004; González-Recio et al., 2004, 2005, 2006).

Australia implemented a multitrait model for genetic evaluation of fertility that encompasses onset of estrus, becoming pregnant, and carrying a pregnancy to term. The model includes calving interval (CVI) as a primary trait, lactation length (LL), days from calving to first service (CFS), first nonreturn rate (FNRR), and conception rate as correlated traits (Haile-Mariam et al., 2013). Although the fertility breeding goal in Australia is conception within $6 \mathrm{wk}$ of the start of the mating season (6WC; Morton, 2004), CVI has been used as the main fertility trait because of lack of pregnancy records in the majority of herds. A linear transformation converts the CVI EBV from the multitrait model to a trait that approximates $6 \mathrm{WC}$ and assumes that the correlation between these traits is one. Recent studies have shown that the genetic correlation between these traits is less than one (Haile-Mariam et al., 2013). Therefore, a trait that uses information from multiple data sources may have a higher correlation with $6 \mathrm{WC}$.

Data on $6 \mathrm{WC}$ is difficult to obtain on all cows without routine and extensive pregnancy testing. However, it is possible to calculate a trait that is close to $6 \mathrm{WC}$ using data from a variety of sources. Here, we define a trait, called CR42, which is defined as the probability of calving after being mated within a period of time that is $42 \mathrm{~d}$ (length of 2 normal reproductive cycles) from the beginning of the mating period. Trait CR42 is derived from pregnancy status, interval from calving to first mating, and calving interval data, and replaces pregnancy rate in the current index. Furthermore, CR42 becomes the main trait to be evaluated, instead of CVI. The CR42 is thought to be closer than CVI to the Australian fertility breeding goal, 6WC. Around $50 \%$ of herds in the Australian dairy industry follow a seasonal system, and it is therefore important to ensure that cows get pregnant within 6 wk $(2$ reproductive cycles) after the mating season begins. This is also valid for year-round calving systems, where the breeding goal may be viewed as getting pregnant within 2 cycles after first fertile estrus. Considerable efforts have been made over the last decade to collect more mating and pregnancy data for genetic evaluation purposes; it is therefore relevant to assess whether the main fertility trait can be changed from CVI to a trait closer to the breeding goal.
We considered a second strategy to increase the rate of genetic gain in fertility, which was including type and yield traits as early indicators of female fertility. Type traits have previously been found to be associated with female fertility. For instance, Pérez-Cabal et al. (2006) reported that impaired fertility was associated with poor scores for foot and legs traits. Type traits associated with energy balance, such as angularity or BCS, have been already shown to be genetically correlated with fertility traits (Pryce et al., 2000). Adding an indicator of energy status at a particular time during lactation could therefore improve the accuracy of prediction of female fertility.

The objectives of this study were to (1) test whether replacing CVI with CR42 as the primary selection criteria increased the correlation between the selection index and the breeding goal, and (2) to investigate whether the addition of type and yield traits in a combined energy status-fertility multitrait model increased the reliability of the CR42 EBV.

\section{MATERIALS AND METHODS}

\section{Data}

Two main data sets of first-parity Holstein-Friesian cows were used to estimate genetic parameters. The first data set comprised reproductive and milk yield traits, and the second data set included type traits on a subset of cows with reproductive data.

The traits analyzed were CVI, LL, CFS, FNRR, CR42, age at first calving (AFC), trait deviations for milk yield (305-d milk, fat, and protein yield), SCS, and 12 type traits: overall type, udder texture (UDTEX), bone quality (BNE), angularity (ANGUL), muzzle width (MUZW), pin width (PNW), pin set, mammary system (MAMM), body depth (BD), foot angle (FA), udder depth, and BCS. All the data used for this study were extracted from the Australian Dairy Herd Improvement Scheme (ADHIS) database. A detailed description of the data used for this study is given by Haile-Mariam et al. (2014). Briefly, data from herds with at least 100 calving records between January 1999 and December 2010 were selected. Bulls used in AI programs with at least 5 daughters were included in this study. Additionally, the study was restricted to firstparity cows that calved between 18 and 38 mo of age. To be valid, CVI had to have a minimum length of 290 d; CVI between 500 and $762 \mathrm{~d}$ were set at a maximum of $501 \mathrm{~d}$ to minimize the influence of extremely long intervals on both the variance and mean. In addition, cows that were recorded as culled for fertility in the ADHIS database were assigned a value for CVI that 
Table 1. Summary statistics of daughter trait deviations used as phenotypes in this study

\begin{tabular}{|c|c|c|c|c|c|c|}
\hline Trait $^{1}$ & $\mathrm{~N}$ & Mean & $\mathrm{SD}$ & Mode & 25th Quantile & 75th Quantile \\
\hline Milk (L) & 164,318 & 3,943 & 607.84 & 3,861 & 3.560 & 4,320 \\
\hline Fat (kg) & 164,318 & 140 & 21.038 & 139 & 127.0 & 153 \\
\hline Protein (kg) & 164,318 & 111 & 17.52 & 112 & 100.0 & 122 \\
\hline $\mathrm{SCC}$ & 154,301 & 180 & 25.51 & 177 & 166.191 & 194 \\
\hline Angularity & 92,012 & 7.39 & 1.03 & 7.49 & 6.77 & 8.08 \\
\hline $\mathrm{BCS}$ & 82,125 & 4.74 & 0.49 & 4.73 & 4.47 & 4.97 \\
\hline Body depth & 92,005 & 7.21 & 1.05 & 7.28 & 6.58 & 7.88 \\
\hline Bone & 92,012 & 6.99 & 1.17 & 7.28 & 6.27 & 7.81 \\
\hline Foot angle & 92,005 & 5.78 & 0.91 & 5.69 & 5.21 & 6.39 \\
\hline MAMM & 91,746 & 10.98 & 1.45 & 11.16 & 10.11 & 11.97 \\
\hline MUZW & 92,012 & 6.64 & 0.97 & 6.82 & 6.07 & 7.28 \\
\hline Overall type & 92,012 & 10.70 & 1.40 & 10.57 & 10.00 & 11.59 \\
\hline Pin width & 92,005 & 7.51 & 1.08 & 7.65 & 6.87 & 8.22 \\
\hline Pin set & 92,005 & 5.61 & 1.18 & 5.82 & 4.90 & 6.33 \\
\hline Udder depth & 92,005 & 7.58 & 1.09 & 7.57 & 6.92 & 8.24 \\
\hline Udder texture & 92,012 & 6.60 & 1.13 & 6.75 & 5.92 & 7.36 \\
\hline Calving interval (d) & 130,542 & 399 & 51.30 & 501 & 363.0 & 422 \\
\hline CFS (d) & 67,578 & 87 & 30.82 & 80 & 70.00 & 99 \\
\hline FNRR $(\%)$ & 64,540 & 55 & 49.73 & 100 & - & - \\
\hline LL (d) & 128,093 & 351 & 54.70 & 451 & 309.0 & 382 \\
\hline CR42 (\%) & 73,394 & 54 & 49.80 & 100 & - & - \\
\hline
\end{tabular}

${ }^{1} \mathrm{CFS}=$ day from calving to first service; CR $42=$ conception rate at d 42 after starting of the mating season; $\mathrm{FNRR}=$ nonreturn rate at first service; $\mathrm{LL}=$ lactation length; MAMM = mammary system; MUZW = muzzle width.

was $3 \mathrm{~d}$ longer than the cow with the longest CVI in the herd-year-season group (contemporary group). In the data used for the current study, about $14.2 \%$ of the cows that did not have a subsequent calving record were included with penalized CVI.

Then, CR42 was calculated using pregnancy status after the mating season begins (assuming a voluntary waiting period of $60 \mathrm{~d}$ ), coded as pregnant (1) or nonpregnant (0) at d 45 after calving (to allow for a possible 1-2 d variation in each reproductive cycle, 42 +3 d). If pregnancy data were not available, CR42 was calculated from CVI and CFS if the 2 measurements were available, which allows the determination of whether the cow was pregnant after $45 \mathrm{~d}$ after the first mating (assuming $282 \mathrm{~d}$ as the pregnancy length). Otherwise, CR42 was set as missing. A summary of the number of records and number of sires with progeny in addition to means and standard deviations of the traits is shown in Table 1.

The second data set used in this study included type scores carried out by Holstein Australia classifiers once during the first lactation. The type data used is described by Haile-Mariam et al. (2014). Briefly, the data comprised linear scores on a scale of 1 to 9 and composite type traits scored on a scale of 1 to 16 . The type data of about 92,000 cows that were classified between 1999 and 2012 were used. Days in milk at the time of classification were from 1 to $365 \mathrm{~d}$. Cows that calved for the first time after 38 mo of age and those classified after 49 mo of age were excluded. The type traits selected for this study are those known to influence economic traits.

Trait deviations for cows were obtained by adjusting for herd-year-season, month of calving (as cross classified), and age at calving (as a covariate). The final edited data set comprised 164,318 cow records sired by 4,766 bulls. A total of 11,593 animals were present in the pedigree file.

\section{Principal Components Analysis}

To investigate the relationship between type traits (n $=12$ ) and fertility traits $(\mathrm{n}=5)$, a principal component analysis (PCA; Pearson, 1901) on the trait deviations was implemented using pca3d package in $\mathrm{R}$ (Weiner, 2013) to form a cluster of traits related to energy status and fertility. The PCA is a multivariate dimension reduction technique that transforms a set of observed variables $\left(y_{1}, y_{2}, \ldots, y_{n}\right)$ to a new coordinate system of $p(p<n)$ variables $\left(x_{1}, x_{2}, \ldots, x_{p}\right)$ to synthesize the amount of information in the data using orthogonal linear transformation. Principal component extraction is carried out in such a way as to successively maximize the amount of original variance in the data explained using a new variable derived from several traits.

The $j$ th principal component is represented by the linear combination of the observed variables:

$$
x_{j}=\sum_{i=1}^{n} \alpha_{i j} y_{i}
$$


where the coefficients $\alpha_{i j}$ are the elements of the eigenvector of the (co)variance (or correlation) matrix of observed variables corresponding to the $j$ th eigenvalue.

The coordinates of the traits in the first 5 factors from the PCA were evaluated for clustering type traits with fertility. Additionally, traits that have been traditionally related to energy balance, such as ANGUL or BCS, were examined to search for any possible cluster between these and other traits. Those traits that clustered together were then included in an independent PCA, keeping the first eigenvector as a new energy status indicator trait (ESt). Although we would have preferred to use repeated type measurements through lactation to calculate energy status as a dynamic trait, type traits are only measured once in lactation. However, the users of the index (i.e., farmers) are likely to recognize this type composite as traits that they would associate with the energy status of the cow, which is why we have referred to the composite as energy status at a given time during lactation. Furthermore, milk, fat, and protein trait deviations were also added to this cluster in a second PCA where the first and second eigenvector were combined to form an energy status indicator trait (ESty) accounting for type and yield.

\section{Multivariate Genetic Analyses}

Bayesian trivariate models were fitted to analyze the yield, type, and fertility traits involved in this study. Data on all individuals is $\mathbf{Y}$, with $\mathbf{y}_{i}=\left(y_{i 1}, y_{i 2}, y_{i 3}\right)$ being the vector of phenotypic observations of animal $i$ for each trait $k(k=1,2,3)$. The sampling model was

$$
\mathbf{Y} \mid \boldsymbol{\beta}, \mathbf{s}, \mathbf{R} \sim N(\mathbf{X} \boldsymbol{\beta}+\mathbf{Z} \mathbf{s}, \mathbf{R} \otimes \mathbf{I}),
$$

where

$$
\mathbf{R}=\left[\begin{array}{ccc}
\sigma_{e 1}^{2} & \sigma_{e 12} & \sigma_{e 13} \\
\sigma_{e 12} & \sigma_{e 2}^{2} & \sigma_{e 23} \\
\sigma_{e 31} & \sigma_{e 32} & \sigma_{e 3}^{2}
\end{array}\right]
$$

is the residual covariance matrix between the 3 traits, and $\mathbf{I}$ is an identity matrix of an order equal to the number of records in the data set. The systematic effects $(\boldsymbol{\beta})$ in the preceding model were regression coefficients with a quadratic term on the age of first calving of the cow and the contemporary group of herd-year-seasonage of first calving, for fertility and milk yield traits. For type traits herd-classifier-round effect instead of herd-year-season-age of first calving effect were fitted. The additive genetic effect of the sire (s) was included in the model and was assumed to be distributed as $\mathbf{s}$ $\mathbf{G} \sim N(\mathbf{0}, \mathbf{G} \otimes \mathbf{A})$ a priori, where

$$
\mathbf{G}=\left[\begin{array}{ccc}
\sigma_{s 1}^{2} & \sigma_{s 12} & \sigma_{s 13} \\
\sigma_{s 12} & \sigma_{s 2}^{2} & \sigma_{s 23} \\
\sigma_{s 31} & \sigma_{s 32} & \sigma_{s 3}^{2}
\end{array}\right]
$$

is the $3 \times 3$ (co)variance matrices for sire additive genetic effect, and $\mathbf{A}$ is the additive relationship matrix in the absence of female pedigree (Henderson, 1976). Then, $\mathbf{X}$ and $\mathbf{Z}$ were the corresponding incidence matrices of systematic and additive genetic sire effect. Independent inverse Wishart prior distributions were assigned to matrices $\mathbf{G}$ and $\mathbf{R}$. Bounded uniform priors $U(-9999,9999)$ were assigned to systematic effects.

The posterior distribution is given by

$$
p(\boldsymbol{\beta}, \mathbf{s} \mid \mathbf{Y}) \propto p(\mathbf{Y} \mid \boldsymbol{\beta}, \mathbf{s}) p(\mathbf{s} \mid \mathbf{G}) p(\mathbf{G}) p(\mathbf{e} \mid \mathbf{R}) p(\mathbf{R}),
$$

where

$$
p(\mathbf{Y} \mid \boldsymbol{\beta}, \mathbf{s}) \propto \prod_{i=1}^{N}|\mathbf{R}|^{1 / 2} \exp \left\{\frac{1}{2}\left[\mathbf{y}_{i}-\mathrm{E}\left(\mathbf{y}_{i}\right)\right]^{\prime} \mathbf{R}^{-1}\left[\mathbf{y}_{i}-\mathrm{E}\left(\mathbf{y}_{i}\right)\right]\right\} .
$$

Above, $\mathrm{E}\left(\mathbf{y}_{i}\right)$ is the expectation of $\mathbf{y}_{i}$. Adopting a threshold model for FNRR and CR42 (Gianola, 1982; Gianola and Foulley, 1983), and regarding their unobserved liability $\left(\boldsymbol{\lambda}=\mathbf{y}_{k}\right)$ as a normal trait, the augmented posterior distributions are as described above, with the following additional specifications.

The liabilities are less or greater than a threshold $(t)$ depending on the observed phenotype

$$
y\left\{\begin{array}{l}
=0 \text { if } \lambda<t \\
=1 \text { if } \lambda>t
\end{array}(t \text { was arbitrarily set to } 0) .\right.
$$

Furthermore, the residual variance for the categorical traits was set to one, and a conditional inverted Wishart distribution is used for sampling $\mathbf{R}$ as described by Korsgaard et al. (2003). The residual covariance between both binary traits was restricted to zero under this specification.

Analyses were performed using TM software (Legarra et al., 2008). Convergence was visually checked from the traces and the stability (deviation lower than 0.05) of the running means during at least 50,000 iterations. Because trivariate analyses were performed, some pairs of traits were analyzed more than once. In these cases, special attention was taken to the posterior distributions. Convergence and posterior densities were 
visually tested. In all cases, posterior distributions from different analyses overlapped to a large extent. To account for all available information, posterior means and standard deviations were calculated using all samples available, assuming they were representative of the same posterior distribution.

\section{Combined Type Fertility Index}

Using the results from the PCA, and estimated genetic correlations, a subset of type traits were selected. Type traits that showed some clustering with fertility traits, and those with genetic correlations above 0.50 (in absolute value), were selected. These traits were used to complement the current traits in the multitrait fertility model used in Australia, after substituting CR by CR42 (CVI, LL, CFS, FNNR, CR42). The accuracy of several fertility indices (adding traits sequentially according to larger increase in the accuracy of the index) were calculated using software developed by Van der Werf (http://www-personal.une.edu.au/ jvanderw/ software.htm), following selection index theory (Hazel, 1943). The selection index model was designed to reflect the current practices of the Australian Dairy Herd Improvement Scheme, with regard to the average number of daughters with records for each trait (Table 2). The genetic and phenotypic (co)variance components used in the model were estimated using the multitrait analyses described earlier. The vector of index weights (b) was derived as $\mathbf{b}=\mathbf{P}^{-1} \mathbf{G}$, where $\mathbf{P}$ is a covariance matrix of dimensions $m \times m$ for the $m$ traits in the selection criterion. This matrix is calculated using a combination of phenotypic and genetic (co)variances and weighted according to the number of records that are used. As the accuracy of EBV increases with the addition of more information from other traits or additional records, the elements in the $\mathbf{P}$ matrix become closer to genetic (co)variances. Matrix $\mathbf{G}$ is the $m \times$ 1 matrix of genetic covariances between the $m$ traits in the selection criterion and the selection objective (CR42 in our case).

\section{Reliabilities}

Additionally, we studied the increase in reliability produced by including the new traits in the current multitrait model, used as a benchmark. The options we considered were the inclusion in the model of

(1) type traits that cluster in the PCA or present high genetic correlation with CR42;

(2) ESt as an indicator traits of energy status derived from type trait; and
(3) ESty as an indicator of energy status derived from type traits in ESt plus milk, fat, and protein yields.

Multitrait BLUP models were then run for each scenario to obtain the EBV reliabilities (REL) for CR42, calculated as

$$
\mathrm{REL}=1-\frac{\mathrm{PEV}}{\sigma_{s}^{2}},
$$

where PEV is the prediction error variance of the CR42 EBV estimated using ASREML (Gilmour et al., 2009), and $\sigma_{s}^{2}$ is the respective additive genetic sire variance. The reliabilities of CR42 EBV were plotted as a function of number of daughters with type and pregnancy test records in a 2-D, heat-colored plot using the filled. contour function in the $\mathrm{R}$ graphics package (Murrell, 2005) using the Nadaraya-Watson nonparametric estimator (Nadaraya, 1964; Watson, 1964).

\section{RESULTS AND DISCUSSION}

\section{Use of CR42 as Primary Fertility Trait}

In the current Australian genetic evaluation of female fertility, the primary trait is CVI. Here, a new trait, CR42, calculated from a combination of calving dates, mating, and pregnancy data is proposed to become the main trait in Australian fertility genetic evaluations. Trait CR42 showed similar heritabilities to CVI in this study (Table 5). However, genetic correlation between CR42 and the breeding objective (6WC) was effectively 1 in a pilot study based on data of about 200,000 cows (unpublished data), whereas genetic correlation of

Table 2. Eigenvalue and percentage of variance explained by each principal component

\begin{tabular}{lccc}
\hline Vector & Eigenvalue & $\begin{array}{c}\text { Percentage } \\
\text { of variance }\end{array}$ & $\begin{array}{c}\text { Cumulative } \\
\text { percentage } \\
\text { of variance }\end{array}$ \\
\hline 1 & 2.376 & 17.0 & 17.0 \\
2 & 1.765 & 12.6 & 29.6 \\
3 & 1.700 & 12.1 & 41.7 \\
4 & 1.237 & 8.8 & 50.5 \\
5 & 1.027 & 7.3 & 57.9 \\
6 & 0.977 & 7.0 & 64.9 \\
7 & 0.919 & 6.6 & 71.4 \\
8 & 0.870 & 6.2 & 77.6 \\
9 & 0.720 & 5.1 & 82.8 \\
10 & 0.689 & 4.9 & 87.7 \\
11 & 0.606 & 4.3 & 92.0 \\
12 & 0.412 & 2.9 & 95.0 \\
13 & 0.388 & 2.8 & 97.8 \\
14 & 0.315 & 2.2 & 100.0 \\
\hline
\end{tabular}


CVI and $6 \mathrm{WC}$ was -0.90 . Previous studies have also reported genetic correlation estimates different from 1 $(-0.65)$ between CVI and 6WC (Haile-Mariam et al., 2004).

Currently, ADHIS implements fertility genetic evaluation using a multitrait model to predict CVI. The final step is a linear conversion of CVI breeding values to a trait similar to CR42. In practice, this means that it is assumed that the correlation between CVI and CR42 is one. In fact, the genetic correlation between CR42 and CVI was high but less than 1 ( $0.90 \pm 0.03$; in absolute value), suggesting that they are not the same traits. The genetic effect of the change of the main selection criteria trait is expected to be small. Also, CR42 is highly correlated with LL $(0.91 \pm 0.03$; in absolute value) because most cows with longer-than-average lactation are in general poor in fertility.

The reason for using CVI up to now is the lack of adequate data on conception rate or information from which a measure similar to 42 -d in-calf rate can be derived. The Australian dairy industry has substantially increased the amount of fertility data used in genetic evaluations, by capturing data from computerized recording software that did not automatically provide data downloads available to the national database.

Now enough justification exists to move the selection criteria trait to be closer to the breeding objective trait. Thus, we propose that CR42 should replace linearly transformed CVI as the primary fertility trait for evaluating fertility breeding values in Australia.

\section{PCA}

Initially, all fertility and type traits were included in preliminary PCA. The variance explained by each component in the PCA is shown in Table 2. The first component explained $17 \%$ of the total variance, and the first 4 components together explained half of the total variability. Principal component (PC)1, PC5, and PC6 explained almost $31 \%$ of the variability. Components from 6th to 14th explained less than $7 \%$ of the total variance each. The coordinates of traits in each of the first $6 \mathrm{PC}$ are shown in Table 3. Traits ANGUL, BNE, BCS, UDTEX, and MAMM are more strongly associated with $\mathrm{PC}$; this group appears to contain traits that have been traditionally related to energy balance and body reserve mobilization. However, because they were only measured once in lactation, it was not possible to evaluate such a dynamic trait (energy balance) but still provides an indication of this. Therefore, this cluster of traits was selected for further analyses and referred to as an energy status indicator. Trait MAMM was excluded because it is already a composite trait. The direction of selection on MAMM, as part of an energy status composite, could impair the favorable response to selection achieved in mammary system, which may be important for mastitis resistance and longevity. The mammary system composite is associated with larger milk yield and better udder conformation (Vollema, 1998; Pérez-Cabal and Alenda, 2002), and because of its association with milk yield, logically it is also unfavorably associated with fertility.

Fertility traits seemed to cluster in PC3, with weights close to 0.90 for CVI and CR42 and 0.25 for AFC. The previous primary fertility trait (CVI) and its proposed replacement (CR42) clustered together, with very similar coordinates (although opposite sign) in the first $6 \mathrm{PC}$ because both traits are highly phenotypically correlated and the effect of replacing one by the other is not expected to be large. In general, weak associa-

Table 3. Coordinate of each trait in each of the first 6 principal components (PC)

\begin{tabular}{lrrrrrr}
\hline Trait $^{1}$ & PC1 & PC2 & PC3 & PC4 & PC5 & PC6 \\
\hline Variance (\%) & \multicolumn{1}{c}{17} & 13 & 12 & 9 & 7 & 7 \\
AFC & 0.07 & 0.05 & 0.25 & 0.14 & 0.33 & -0.76 \\
UDTEX & 0.77 & -0.07 & -0.09 & 0.00 & -0.01 & 0.07 \\
BNE & 0.63 & -0.33 & -0.10 & -0.27 & 0.05 & -0.01 \\
ANGUL & 0.70 & 0.37 & -0.06 & -0.18 & -0.05 & 0.05 \\
MUZW & -0.01 & 0.65 & 0.05 & 0.19 & -0.00 & -0.10 \\
PNW & 0.31 & 0.26 & 0.00 & 0.27 & 0.43 & -0.10 \\
Pin set & -0.10 & 0.05 & 0.00 & -0.07 & 0.83 & 0.39 \\
MAMM & 0.65 & 0.03 & -0.09 & 0.46 & -0.11 & 0.04 \\
BD & 0.28 & 0.76 & 0.01 & -0.15 & -0.12 & 0.04 \\
FA & -0.07 & 0.13 & 0.04 & 0.51 & -0.04 & 0.43 \\
UD & 0.14 & -0.45 & -0.03 & 0.67 & -0.03 & -0.05 \\
BCS & -0.45 & 0.46 & 0.08 & 0.23 & -0.14 & -0.02 \\
CVI & 0.15 & -0.07 & 0.89 & -0.04 & -0.05 & 0.12 \\
CR42 & -0.14 & 0.07 & -0.90 & 0.03 & 0.04 & -0.09 \\
\hline
\end{tabular}

${ }^{1} \mathrm{AFC}=$ age at first calving; $\mathrm{ANGUL}=$ angularity; $\mathrm{BD}=$ body depth; $\mathrm{BNE}=$ bone quality; $\mathrm{CVI}=$ calving to first service; CR $42=$ conception rate at d 42 after starting of the mating season; FA $=$ foot angle; MUZW = muzzle width; MAMM = mammary system; PNW = pin width; UD = udder depth; UDTEX = udder texture. 
tions between fertility and type traits were observed in this PCA. Type traits with larger weights on PC3 were UDTEX, BNE, MAMM, and BCS, with values closer to 0.10, which were low but larger among type traits. The PC5 showed a cluster of PNW and pin set with AFC. The PC6 showed larger weights for AFC, pin set, and FA, with weights around 0.10 for MUZW, PNW, CVI, and CR42. All the traits that showed any level of association with fertility were then selected as candidates in the construction of the type-fertility selection indices. This filtering step aimed not to be too restrictive so that all these traits (FA, PNW, pin set, MUZW, SCC, and BCS) had an opportunity to be included in the index.

Then, a new PCA was performed only with traits in the energy status cluster from PC1. The cumulative variance explained for each PC is shown in Table 4 . The first PC explained almost half $(49 \%)$ of the total variance, with weights of $30,29,23$, and $18 \%$ for UDTEX, BNE, ANGUL, and BCS, respectively. When yield traits were also included in the energy status PCA, the first PC explained a lower percentage $(36 \%)$ of the total variance, with production traits having $84 \%$ of the weight in this PC $(30,23$, and $32 \%$ for milk, fat, and protein yield, respectively).

\section{Genetic Correlations of Type and Fertility Traits}

The posterior mean estimates of the genetic correlations, and their posterior standard deviations, are shown in Table 5 (we presented posterior standard deviations because none of the posterior distributions showed asymmetry). Genetic correlations among fertility traits were strong ( $>0.65$, in absolute value), except between LL and CFS (0.55 \pm 0.09$)$ and CFS and FNRR (0.31 \pm 0.11 ). These estimates are similar to those previously reported in different data sets of the same population by Haile-Mariam et al. (2004) and Haile-Mariam et al. (2013) and also in the same range and direction to previous estimates in different populations (Weigel and Rekaya, 2000; Veerkamp et al., 2001; Kadarmideen et al., 2003; González-Recio and Alenda, 2005).

Genetic correlations of fertility traits with type traits were low to moderate, although there were 2 exceptions: (1) ANGUL, which had genetic correlations, expressed in absolute values, between 0.60 and 0.70 with CR42, LL, or FNRR; and (2) UDTEX, which had a genetic correlation of -0.53 with CR42. Absolute value correlations of less than 0.30 were estimated between fertility traits and FA, PNW, pin set, MUZW, SCC, or BCS. Although these traits clustered phenotypically with CR42, their genetic correlations were not large. Body depth and overall type showed low to moderate
Table 4. Cumulative variance (\%) explained by each principal component $(\mathrm{PC})$ for the 2 energy-status clusterings ${ }^{1}$

\begin{tabular}{lcc}
\hline Item & ESt & ESty \\
\hline PC1 & 49 & 36 \\
PC2 & 70 & 63 \\
PC3 & 86 & 74 \\
PC4 & 100 & 84 \\
PC5 & - & 91 \\
PC6 & - & 98 \\
PC7 & - & 100 \\
\hline
\end{tabular}

${ }^{1}$ ESt contains angularity, BCS, bone, and udder texture; ESty contains in addition milk, fat, and protein yields.

correlation $(-0.40 \pm 0.075)$ with $\mathrm{CR} 42$, and similar with other fertility traits. The posterior mean of the genetic correlation estimate between ANGUL and UDTEX was $0.64( \pm 0.04)$ and between $\mathrm{BD}$ and overall type was $0.59( \pm 0.03)$. Angularity had strong genetic correlations with BD $(0.89 \pm 0.01)$ and UDTEX $(0.64$ $\pm 0.04)$. Genetic correlations between type and fertility traits were in agreement with previous studies (Pryce et al., 2000; Pérez-Cabal et al., 2006). Rump traits such as pin set or PNW had genetic correlations with fertility of less than 0.30 (expressed as absolute values), despite the generalized perception that these traits improve fertility. These results suggest that to improve fertility, the best selection criteria are fertility traits. Therefore, the goal of recording schemes for genetic evaluation purposes should be to get a comprehensive data set of a broad range of fertility traits to cover the basic requirements of good fertility, that is, resumption of estrus to establish a viable, full-term pregnancy.

In general, the type traits that were more strongly related to fertility were associated with mobilization of body reserves (e.g., ANGUL, BCS, BNE, or UDTEX), which is in accordance with the established theory that being in negative energy balance impairs fertility in dairy cattle (Butler et al., 1981; Butler and Smith, 1989). Cows begin to have normal estrous cycles 34 d after calving (Hafez, 1980), a period that coincides with the peak in milk production. During this time, energy demands are quite high due to the requirements needed for high production, which leads to a mismatch between energy availability and requirements and consequently negative energy balance. Because reproductive function is secondary, ovulation does not occur until the energy balance begins returning toward zero, leading to frequent fails in conception in current dairy populations. The energy status type composite (ESt; $\left.\mathrm{h}^{2}=0.25 \pm 0.02\right)$ has genetic correlations of $0.41,0.45$, and -0.49 with CVI, LL, and CR42, and close to 0.25 (in absolute values) with CFS and FNRR. However, the energy status composite from milk and type has an even larger heritability $(0.28 \pm 0.01)$ but lower genetic 


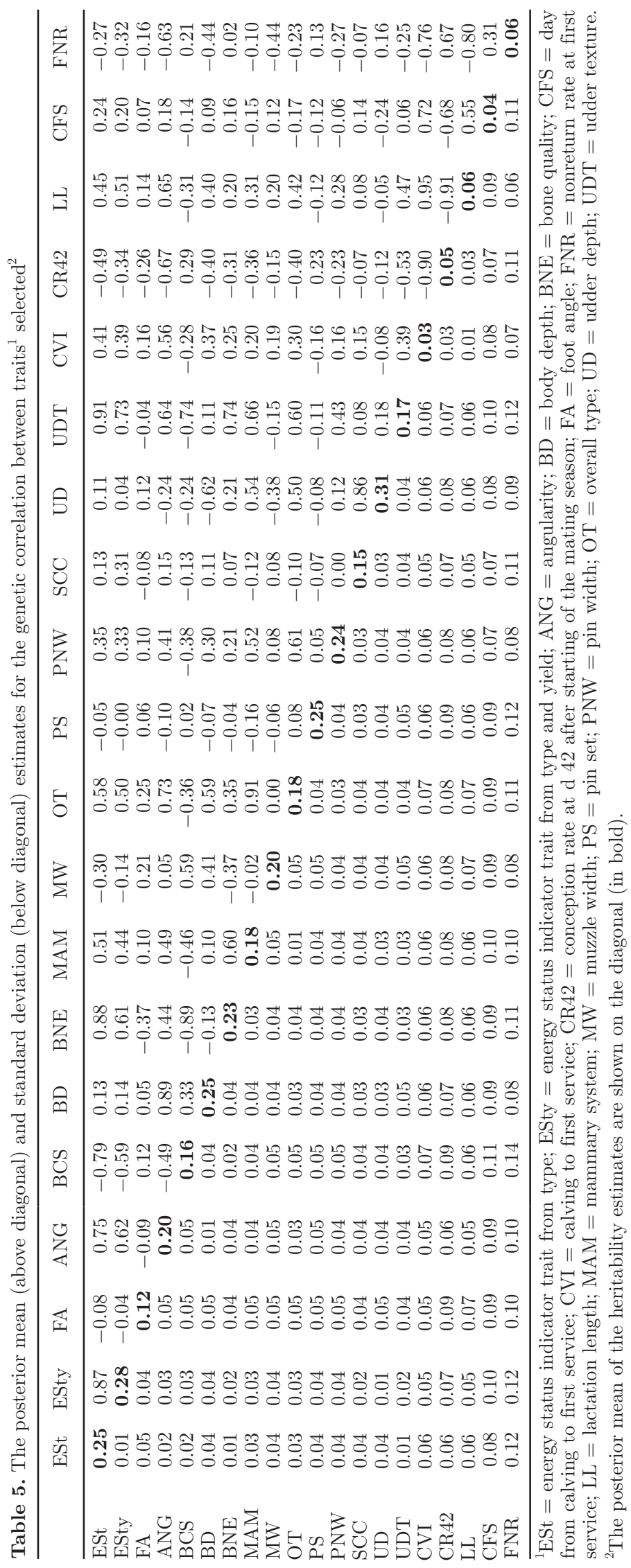


Table 6. Accuracy of several selection indices and expected genetic gain on the conception rate at $42 \mathrm{~d}$ after the beginning of the mating period

\begin{tabular}{llcc}
\hline Index abbreviation $^{1}$ & Traits $^{2}$ & $\begin{array}{c}\text { Reliability } \\
(\%)\end{array}$ & $\begin{array}{c}\text { Expected genetic gain } \\
\text { CR42 (SD/yr) }\end{array}$ \\
\hline Main trait & CR42 & 39 & 0.063 \\
Previous main trait & CVI & 26 & 0.051 \\
IF & CVI, LL, CFS, FNRR, CR42 & 45 & 0.071 \\
IFT1 & IF + ANGUL & 57 & 0.079 \\
IFT2 & IF + ANGUL + FA & 59 & 0.079 \\
IFT3 & IF + ANGUL + FA + pin set & 60 & 0.080 \\
\hline
\end{tabular}

${ }^{1} \mathrm{CVI}=$ calving interval; IF $=$ benchmark index based on fertility traits currently used in Australia; IFT1 = index based on IF and type traits (angularity); IFT2 = index based on IF and type traits (angularity and foot angle); IFT3 = index based on IF and type traits (angularity, foot angle, and pin set).

${ }^{2} \mathrm{CR} 42$ = conception rate at d 42 after starting of the mating season; LL = lactation length; CFS = day from calving to first service; FNRR = nonreturn rate at first service; ANGUL = angularity; FA = foot angle.

correlations: $0.27,0.44$, and -0.09 with CVI, LL, and CR42, respectively.

Ideally, traits that can serve as an indicator of energy status, such as BCS, should be evaluated multiple times across lactation, so that dynamic changes in energy status could be calculated and so that ultimately a trait that is closer to energy balance can be defined. However, in our data, BCS (and all type traits) were only available once per lactation. Pryce and Harris (2006) evaluated the correlation of BCS (measured once per cow) with fertility longitudinally using a random regression sire model applied to New Zealand dairy cattle. They found that the correlation between CR42 and BCS varied between 0.40 and 0.49 across lactation. Surprisingly, the association between BCS and CR42 in New Zealand cattle was stronger in later lactation, that is, much later than conception occurred, implying that energy reserves in later lactation are indicative of fertility performance in early lactation. The implications for our study are that the consistency of the correlation across lactation means that single records of BCS are likely to be adequate for evaluation of the energy status trait.

\section{Combined Type-Fertility Selection Index}

The different type-fertility indices and the theoretical reliabilities are shown in Table 6 . The traits that clustered with CR42 in the PCA (FA, PNW, pin set, MUZW, SCC, and BCS) and those that showed genetic correlation estimates (in absolute value) above 0.50 (ANGUL, UDTEX) were added to an index that included the 5 fertility traits currently used by ADHIS in the routine calculation of fertility breeding values. The fertility index that comprised only the fertility traits currently used in Australia (CVI, LL, CFS, FNRR, and CR42) achieved a reliability of $45 \%$, and the expected genetic gain in CR42 was +0.071 genetic standard deviations per year (i.e., 0.25 standard devia- tion improvement in the index), which is larger than that obtained by CR42 by itself (reliability of $39 \%$ and expected genetic gain of +0.063 genetic standard deviations per year). The addition of type traits increased the reliability of the fertility index and the expected genetic gain in the breeding goal. For example, ANGUL was the type trait with largest weight in the index and which provided the biggest increase in the reliability of the index (57\%). This could be because angularity is also correlated with milk production and therefore also correlated with fertility. The expected genetic gain in the breeding goal when ANGUL was added was +0.079 standard genetic deviations. Trait FA further increased the index reliability by $2 \%$ over the previous one, although no noticeable improvement existed in expected genetic gain. Adding pin set achieved an additional increase of $1 \%$ in the index reliability and resulted in an overall expected genetic gain of +0.080 genetic standard deviations of CR42. PNW, UDTEX, MUZW, $\mathrm{BCS}$, or SCC did not increase the index reliability or the expected genetic gain in the breeding goal. Table 6 also shows that the reliability using only CVI as correlated trait would be lower $(26 \%)$ than those showed above.

\section{EBV Reliabilities}

The reliabilities of CR42 of 3 multitrait BLUP models that are extensions of the current Australian multitrait fertility index were compared for sires with a range of progeny group sizes. The current index was used as a benchmark. Hence, the 3 models included the following traits: (1) ANGUL, FA, pin set, CVI, LL, CFS, FNRR, and CR42; (2) ESt, CVI, LL, CFS, FNRR, and CR42; and (3) ESty, CVI, LL, CFS, FNRR, and CR42.

The increase in EBV reliability from the type-fertility multitrait models, compared with the benchmark model, is shown in Figure 1. The larger relative increase in reliability was observed for sires with 10 to 
Fertility+(ANGUL, Pin Set, FA)

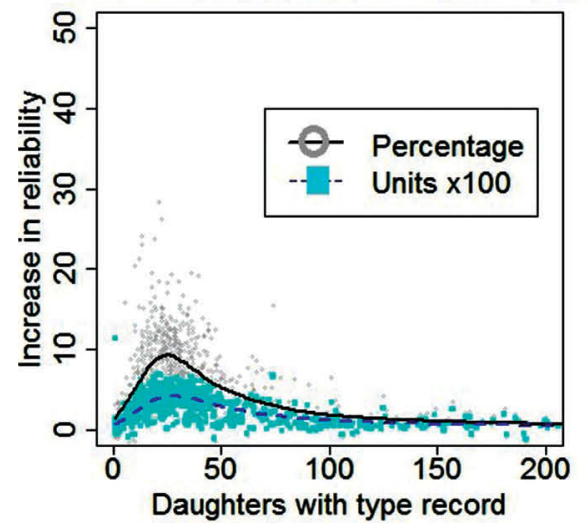

Fertility+(ESt)

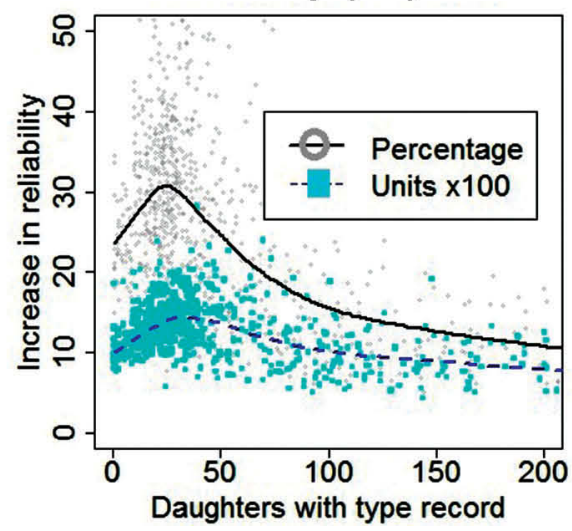

Fertility+(ESty)

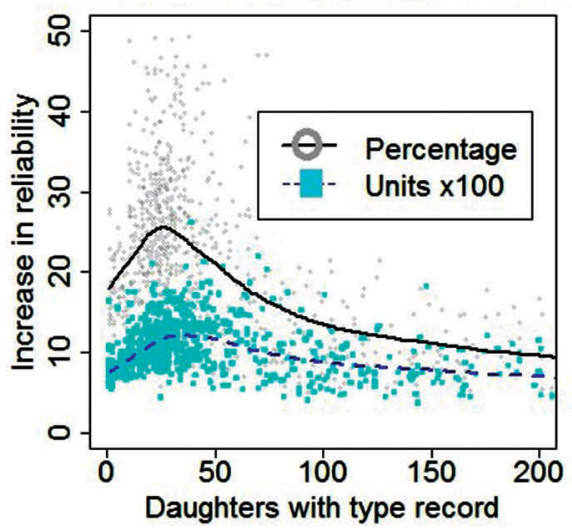

Figure 1. Increase in reliability (units or percentage) with respect to the benchmark model according to the number of daughters with type records for each of the 3 models tested. ANGUL = angularity; FA = foot angle; ESt = energy status indicator trait from type; ESty = energy status indicator trait from type and yield. Color version available online.

50 daughters with type record, where CR42 EBV reliability increased, on average, up to a 10, 30, and $25 \%$ with models 1,2 , and 3 , respectively. A larger overall increase was observed with ESt compared with a model that included the type traits (ANGUL, pin set, and FA). The size of the increase in CR42 reliability from this model was 12 points in reliability (i.e., increased by $30 \%$ ). Trait ESt provided slightly larger reliability (between 0.70 and 2.4 units) than ESty, probably because the PC from the former explained a larger percentage of the phenotypic variation.

As expected, sires with a larger number of daughters with records for CR42 and type had larger reliabilities (Figure 2). For instance, a sire with 40 daughters recorded for CR42 had an average reliability of 54\%; if these daughters also had type data, then the average reliability was $66 \%$. The lower the number of daughters with CR42 phenotype, the larger the contribution of type records to the CR42 reliability. Sires with more than 80 daughters with CR42 records led to the reliability of CR 42 getting close to $80 \%$ on average and did not benefit from daughters with type records. Figure 2 also shows the cost of phenotyping to obtain such reliabilities (assuming a cost of AU\$14 per typed cow, AU \$5 per pregnancy-checked cow, and that breeding decisions are made on only one pregnancy check per lactation). Although the same reliability can be obtained with different combinations of the number of cows with type and pregnancy records, at a given reliability, it is more effective to increase the number of cows with pregnancy records $42 \mathrm{~d}$ after the beginning of the mating period rather than the number of cows with type data. Therefore, efforts to increase the reliability of fertility EBV are most cost effective when directed at obtaining additional pregnancy test data.
The cost of phenotyping and achieved reliabilities must be considered in designing new breeding strategies (González-Recio et al., 2014). If bulls that are part of progeny testing have more than 80 first-crop daughters with a CR42 record, then type traits would not increase the reliability by much. However, until this is a reality and while type records are available for other purposes, their utility in a composite index as an indicator of energy status may assist in increasing the reliability of sire fertility EBV in dairy populations, especially for young sires. Furthermore, type traits will be available only as long as breeders and farmers are interested in getting type assessments.

The collective efforts of breeding schemes worldwide have slowed down the negative trends in fertility around the world, and some countries have even reported a positive trend in some female fertility traits (Pryce et al., 2014). Additional efforts like those described here to increase the reliability of fertility EBV and those described in Byrne et al. (2015) in developing indexes with greater emphasis on fertility may ensure that future genetic trends in female fertility in dairy cattle are favorable.

\section{CONCLUSIONS}

In this study we propose CR42 as the primary selection criteria for improving fertility in Australia and that the multitrait fertility model be extended to include a composite index of type traits, which is an indicator of energy status. The new selection trait is expected to be more correlated with the breeding objective and is also similar to CVI, which is the primary trait in the current multitrait model. The energy status composite, including information from ANGUL, BNE, BCS, 


\section{Reliability of CR42}

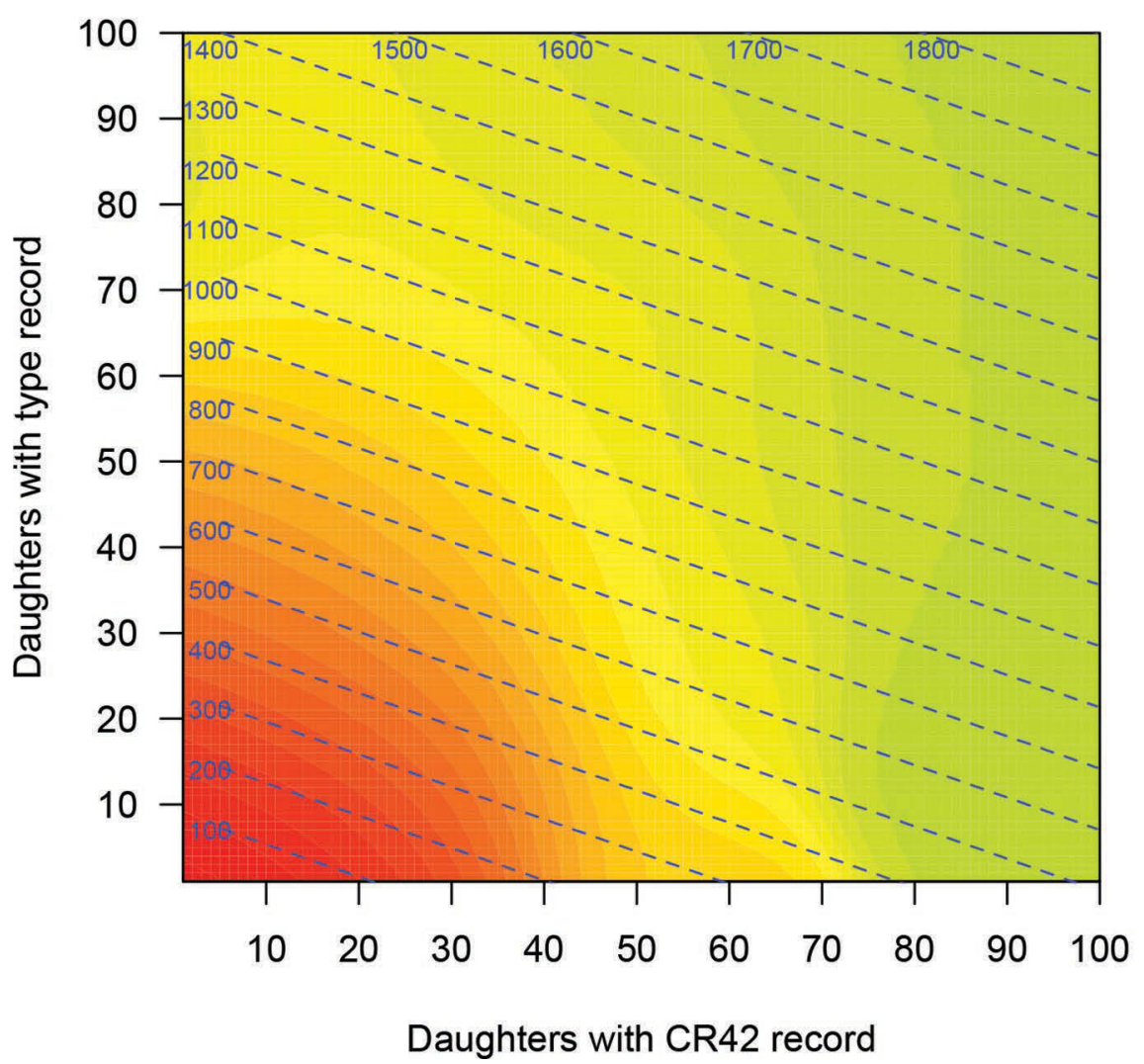

REL
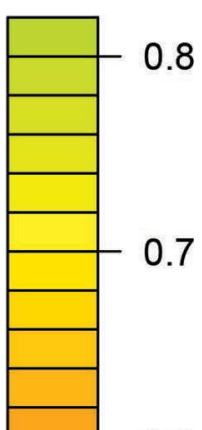

0.6

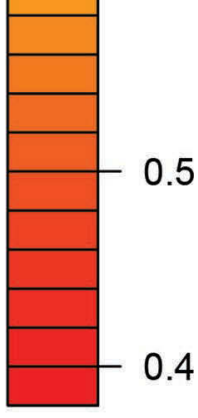

Figure 2. Color map of reliabilities (REL) of conception rate at d 42 after starting of the mating season (CR42) according to the number of daughters with CR42 and type records using a combined energy status (from type traits) and fertility multitrait model. Cost of phenotyping to

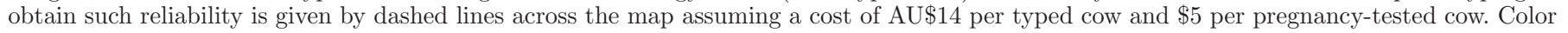
version available online.

and UDTEX, could increase the reliability by $30 \%$ for sires with a lower number of daughters with mating and pregnancy information, usually sires with first-crop daughters. The contribution of energy status-related type traits diminished as more daughters with CR42 information became available. It is important that phenotyping strategies focus on recording additional pregnancy and mating information, or incorporate type records when the number of daughters per sire is lower than 80 .

\section{ACKNOWLEDGMENTS}

The data used for this study were provided by the Australian Dairy Herd Improvement Scheme database (ADHIS, Melbourne, Australia). The authors thank Holstein Australia (Melbourne, Australia) for the type classification data. Dairy Futures Cooperative Research Centre (Melbourne, Australia) is acknowledged for financial support.

\section{REFERENCES}

Bascom, S. S., and J. Young. 1998. A summary of the reason why farmers cull cows. J. Dairy Sci. 81:2299-2305.

Bell, M. J., R. J. Eckard, M. Haile-Mariam, and J. E. Pryce. 2013. The effect of changing cow production and fitness traits on net income and greenhouse gas emissions from Australian dairy systems. J. Dairy Sci. 96:7918-7931.

Butler, W. R., R. W. Everett, and C. E. Coppock. 1981. The relationships between energy balance, milk production, and ovulation in postpartum Holstein cows. J. Anim. Sci. 53:742-748.

Butler, W. R., and R. D. Smith. 1989. Interrelationships between energy balance and postpartum reproductive function in dairy cattle. J. Dairy Sci. 72:767-783.

Byrne, T., D. Martin-Collado, B. Santos, P. Amer, J. Pryce, and M. Axford. 2015. A review of the national breeding objective and selection indexes for the Australian dairy industry. Pages 53-56 in Proc. Assoc. Advance. Anim. Breed. Genet. Lorne, Australia. Association for the Advancement of Animal Breeding and Genetics, Bundoora, VIC, Australia.

Gianola, D. 1982. Theory and analysis of threshold characters. J. Anim. Sci. 54:1079-1096.

Gianola, D., and J. L. Foulley. 1983. Sire evaluation for ordered categorical data with a threshold model. Genet. Sel. Evol. 15:201-224.

Gilmour A. R., B. J. Gogel, B. R. Cullis, and R. Thompson. 2009. ASReml User Guide Release 3.0. VSN Int. Ltd., Hemel Hempstead, UK. 
González-Recio, O., and R. Alenda. 2005. Genetic parameters for female fertility traits and a fertility index in Spanish dairy cattle. J. Dairy Sci. 88:3282-3289.

González-Recio, O., Y. M. Chang, D. Gianola, and K. Weigel. 2005. Number of inseminations to conception in Holstein cows using censored records and time-dependent covariates. J. Dairy Sci. 88:3655-3662.

González-Recio, O., Y. M. Chang, D. Gianola, and K. Weigel. 2006. Comparisons of models using different censoring scenarios for days open in Spanish Holstein cows. Anim. Sci. 82:233-239.

González-Recio, O., M. P. Coffey, and J. E. Pryce. 2014. On the value of the phenotypes in the genomic era. J. Dairy Sci. 97:7905-7915.

González-Recio, O., M. A. Pérez-Cabal, and R. Alenda. 2004. Economic value of female fertility and its relationship with profit in Spanish dairy cattle. J. Dairy Sci. 87:3053-3061.

Groen, A. F., J. Aumann, V. Ducrocq, N. Gengler, J. Soelkner, and E. Strandberg. 1998. Genetic improvement of functional traits in cattle. (GIFT). Interbull Bull. 17:81-82.

Hafez, E. S. E. 1980. Reproduction in Farm Animals. 4th ed. Lea Febiger, Philadelphia, PA.

Haile-Mariam, M., P. J. Bowman, and M. E. Goddard. 2004. Genetic parameters of fertility traits and their genetic correlation with production, type, workability, live weight, survival index and somatic cell count. Aust. J. Agric. Res. 55:77-87.

Haile-Mariam, M., P. J. Bowman, and J. E. Pryce. 2013. Genetic analysis of fertility and predictor traits in Holstein herds with low and high mean calving intervals, and in Jersey. J. Dairy Sci. 96:655-667.

Haile-Mariam, M., O. González-Recio, and J. E. Pryce. 2014. Prediction of liveweight of cows from type traits and its relationship with production and fitness traits. J. Dairy Sci. 97:3173-3189.

Hazel, L. N. 1943. The genetic basis for constructing selection indices. Genetics 28:476-490.

Henderson, C. R. 1976. Inverse of a matrix of relationships due to sires and maternal grandsires in an inbred population. J. Dairy Sci. 59:1585-1588.

Kadarmideen, H. N., R. Thompson, M. P. Coffey, and M. A. Kossaibati. 2003. Genetic parameters and evaluations from single- and multiple-trait analysis of dairy cow fertility and milk production. Livest. Prod. Sci. 81:183-195.

Korsgaard, I., M. S. Lund, D. Sorensen, D. Gianola, P. Madsen, and J. Jensen. 2003. Multivariate Bayesian analyses of Gaussian, right censored Gaussian, ordered categorical and binary traits using Gibbs sampling. Genet. Sel. Evol. 35:159-183.

Legarra, A., L. Varona, and E. López de Maturana. 2008. TM Threshold Model. Accessed Oct. 31, 2008. http://snp.toulouse.inra. $\mathrm{fr} /$ alegarra/manualtm.pdf.
Morton, J. 2004. Determinants of reproductive performance of dairy cows in commercial herds in Australia. PhD Thesis. Univ. Melbourne. Accessed Nov. 11, 2011. http://repository.unimelb.edu. au/10187/1503.

Murrell, P. 2005. R Graphics. Chapman Hall/CRC Press, Boca Raton, FL.

Nadaraya, E. A. 1964. On estimating regression. Theory Probab. Appl. 9:141-142.

Oseni, S., S. Tsuruta, I. Misztal, and R. Rekaya. 2004. Genetic parameters for days open and pregnancy rates in US Holstein using different editing criteria. J. Dairy Sci. 87:4327-4333.

Pearson, K. 1901. On lines and planes of closest fit to systems of points in space. Philosoph. Mag. 211559-572.

Pérez-Cabal, M. A., and R. Alenda. 2002. Genetic relationship between lifetime profit and type traits in Spanish dairy cattle. J. Dairy Sci. 85:3480-3491.

Pérez-Cabal, M. A., C. García, O. González-Recio, and R. Alenda. 2006. Genetic and phenotypic relationships among locomotion type traits, profit, production, longevity, and fertility in Spanish dairy cows. J. Dairy Sci. 89:1776-1783.

Pryce, J. E., M. P. Coffey, and S. Brotherstone. 2000. The genetic relationship between calving interval, body condition score and linear type and management traits registered in Holstein. J. Dairy Sci. 83:2664-2671.

Pryce, J. E., and B. L. Harris. 2006. Genetics of body condition score in New Zealand dairy cows. J. Dairy Sci. 89:4424-4432.

Pryce, J. P., R. Woolaston, D. P. Berry, E. Wall, M. Winters, R. Butler, and M. Shaffer. 2014. World trends in dairy cattle. Abstract 154 in Proc. 10th World Congr. Genet. Appl. Livest. Prod. Vancouver, Canada.

Veerkamp, R. F., E. P. C. Koenen, and G. De Jong. 2001. Genetic correlations among body condition score, yield, and fertility in first-parity cows estimated by random regression models. J. Dairy Sci. 84:2327-2335.

Vollema, A. R. 1998. Longevity of dairy cows: A review of genetic variances and covariances with conformation. Anim. Breed. Abstr. 66:781-802.

Watson, G. S. 1964. Smooth regression analysis. Sankhya A 26:359372.

Weigel, K. A., and R. Rekaya. 2000. Genetic parameters for reproductive traits of Holstein cattle in California and Minnesota. J. Dairy Sci. 83:1072-1080.

Weiner, J. 2013. pca3d: Three dimensional PCA plots. R package version 0.2. Accessed Oct. 26, 2015. http://CRAN.R-project.org/ package $=$ pca3d. 